\title{
AVALIAÇÃO DA UNIVERSALIDADE DAS NORMAS DRIS, M-DRIS E CND ${ }^{(1)}$
}

\author{
Gualter Guenther Costa da Silva ${ }^{(2)}$, Júlio César Lima Neves ${ }^{(3)}$, \\ Víctor Hugo Alvarez V. ${ }^{(3)}$ \& Fernando Palha Leite ${ }^{(4)}$
}

\begin{abstract}
RESUMO
A possibilidade de utilização de normas obtidas em determinado local, para diferentes condições de clima e solo, pode conferir aos métodos de diagnose do estado nutricional um caráter de universalidade. Neste sentido, por meio da aplicação dos métodos: Sistema Integrado de Diagnose e Recomendação (DRIS), DRIS modificado (M-DRIS) e Diagnose da Composição Nutricional (CND) em plantios florestais de Eucalyptus grandis, objetivou-se comparar a diagnose, aplicando-se os métodos, mediante normas específicas ou gerais, em localidades da região Centro-Leste do Estado de Minas Gerais, utilizando diferentes critérios de avaliação. Foram utilizados dados de produtividade e de teores de N, P, K, Ca e Mg nas folhas, referentes a 993 talhões de Eucalyptus grandis plantados no espaçamento $3 \times 2 \mathrm{~m}$, com idades variando de 72 a 153 meses, e abrangendo seis localidades da região estudada. A universalidade das normas foi avaliada, comparando-se a freqüência de diagnoses concordantes (FDC) do potencial de resposta à adubação (PRA), obtida mediante normas específicas ou gerais, considerando os seguintes critérios de avaliação: os nutrientes separadamente; desde todos (5) até nenhum nutriente (0); e apenas para o limitante primário por deficiência (p) ou por excesso (n). O grau de universalidade das normas variou de acordo com o critério adotado para sua avaliação. Em virtude da ausência de universalidade das normas, é preferível a utilização de normas específicas em vez de normas gerais.
\end{abstract}

Termos de indexação: Eucalyptus grandis, diagnose foliar, normas específicas, normas gerais.

\footnotetext{
(1) Parte da Tese de Mestrado do primeiro autor, apresentada ao Departamento de Solos da Universidade Federal de Viçosa - UFV. Financiado pela CAPES e pela CENIBRA Florestal. Recebido para publicação em junho de 2004 e aprovado em agosto de 2005.

(2) Doutorando do Departamento de Solos, Universidade Federal de Viçosa - UFV. CEP 36570-000 Viçosa (MG). Bolsista CNPq. E-mail: gualtergcs@yahoo.com.br

(3) Professor do Departamento de Solos, UFV. Bolsista do CNPq. E-mail: vhav@ufv.br;

${ }^{(4)}$ Pesquisador da CENIBRA Florestal. Ipatinga (MG). E-mail: gualter@zipmail.com.br
} 


\title{
SUMMARY: EVALUATION OF THE UNIVERSALITY OF DRIS, M-DRIS, AND CND NORMS
}

\begin{abstract}
The possibility of using norms obtained at a given site for different climate and soil conditions can confer the methods of diagnosis of nutritional status a universal character. In this sense, the methods Diagnosis and Recommendation Integrated System (DRIS), modified DRIS (M-DRIS), and Compositional Nutrient Diagnosis (CND) were applied in Eucalyptus grandis plantations aiming at comparing the diagnosis established by the methods with either specific or general norms at several sites in the central-eastern region of Minas Gerais State, Brazil, under different evaluation criteria. Yield data and the N, $P, K$, $\mathrm{Ca}$, and $\mathrm{Mg}$ leaf contents from 993 Eucalyptus grandis stands (spacing $3 \times 2 \mathrm{~m}$ ) at ages between 72 and 153 months were used from six sites in the study region. The validity of the universal norm was evaluated by comparing the Frequency of Concordant Diagnosis (FDC) derived from the response potential to fertilization (PRA), obtained by specific or general norms under the following evaluation criteria: nutrients separately; varying from all of them (5) to no nutrient (0); only for the primary limiting nutrient by deficiency ( $p$ ), or by excess (n). The universality degree varied according to the adopted evaluation criterion. Due to the lack of universality of the norms, the use of specific norms instead of general norms is preferable.
\end{abstract}

Index terms: Eucalyptus grandis, leaf diagnosis, specific norms, general norms.

\section{INTRODUÇÃO}

A avaliação do estado nutricional de plantas, por meio da aplicação dos métodos de diagnose foliar, relacionando os teores de nutrientes entre si, temse destacado dos métodos tradicionais de diagnose que consideram os teores dos nutrientes individualmente, como, por exemplo, o do nível crítico. O Sistema Integrado de Diagnose e Recomendação (DRIS), proposto por Beaufils, é o principal método bivariado utilizado. Foi criado com o objetivo de classificar os nutrientes quanto à ordem de limitação ao desenvolvimento e crescimento das plantas (Costa, 1999), independentemente da idade ou órgão da planta a ser amostrado (Bailey et al., 1997). A partir do DRIS, são calculados índices que expressam o equilíbrio relativo dos nutrientes numa planta, por meio da comparação de relações duais (N/P, P/K, K/Ca, Ca/Mg, etc) na amostra, com valores padrões ou normas (Alvarez V. \& Leite, 1999).

A principal premissa para utilização do DRIS refere-se à relativa constância das relações entre nutrientes, (relações duais), comparativamente aos teores de cada nutriente, considerados isoladamente, como também em relação à idade do tecido e seu teor de água (Beaufils, 1973).

Portanto, existe a proposição de que as normas DRIS possam ser obtidas e utilizadas, independentemente do cultivar ou da região (Sumner, 1979; Walworth \& Sumner, 1987; Payne et al., 1990; Wadt, 1996). Também, a literatura registra que, provavelmente, existem espécies para as quais as relações ótimas entre nutrientes são pouco afetadas por condições locais, como solo e clima (Snyder \& Kretschmer, 1988; Snyder et al., 1989); portanto, podem ser utilizados com caráter universal, apesar de ser obtida maior exatidão pelo uso de normas específicas (Escano et al., 1981; Dara et al., 1992; Wortmann et al., 1992; Jones Jr., 1993; Costa, 1999; Reis Jr., 2001; Reis, 2002).

A idéia de universalidade refere-se à possibilidade de utilização de normas DRIS obtidas em determinado local, para diferentes condições de clima e solo (Snyder \& Kretschmer, 1988; Snyder et al., 1989). Parte-se da premissa de que, pelo fato de as relações duais entre nutrientes apresentarem maior constância comparativamente aos seus teores, haveria certa independência no uso do DRIS em relação a fatores reconhecidamente influentes nos teores de nutrientes, como a região de cultivo, a idade e o órgão da planta a ser amostrado, dentre outros.

Além do DRIS, destaca-se, como método bivariado, o DRIS modificado (M-DRIS), e, como método multivariado, o da Diagnose da Composição Nutricional (CND). No M-DRIS (Hallmark et al., 1987), além das relações duais, os teores dos nutrientes também são considerados. O método CND foi desenvolvido por Parent \& Dafir (1992), de acordo com os estudos desenvolvidos por Aitchison (1982), concernentes à análise estatística de dados de composição, com base na obtenção de variáveis multinutrientes $\left(\mathrm{Z}_{\mathrm{i}}\right)$, cada uma delas ponderada pela média geométrica da composição nutricional.

A consistência da interpretação das análises de tecido aumenta à medida que o enfoque univariado (o do nível crítico) é ampliado, de modo a considerar as relações entre nutrientes, dois a dois, ou seja, relações duais (enfoque bivariado) e, assim, progressivamente, as relações ternárias até 
idealmente abranger, mediante enfoque multivariado, toda a estrutura de variação da composição nutricional (Holland, 1966). Portanto, a comparação de métodos de diagnose nutricional, com diferentes procedimentos para o cálculo e interpretação de seus índices, permite avaliar a influência de cada método quanto ao aspecto de universalidade das respectivas normas.

Neste sentido, o presente trabalho foi realizado com os métodos DRIS, M-DRIS e CND, em talhões de florestas de eucalipto, em localidades da região Centro-Leste do Estado de Minas Gerais, tendo como objetivo avaliar a universalidade das normas, comparando a diagnose nutricional realizada com o uso de normas específicas com a de normas gerais.

\section{MATERIAL E MÉTODOS}

Foram utilizados dados de produtividade e teores de N, P, K, Ca e Mg em folhas de Eucalyptus grandis, com idade variando de 72 a 153 meses, plantados no espaçamento de $3 \times 2 \mathrm{~m}$, em seis localidades: Cocais, Piracicaba, Rio Doce, Sabinópolis, Santa Bárbara e Virginópolis, na região Centro-Leste do Estado de Minas Gerais (Quadro 1), perfazendo um total de 993 talhões.

Os dados desses talhões originaram as populações de interesse de estudo, sendo para tanto: (a) estratificadas por localidade: Cocais $(\mathrm{n}=191$ talhões), Piracicaba $(n=201)$, Rio Doce $(n=54)$, Sabinópolis $(\mathrm{n}=198)$, Santa Bárbara $(\mathrm{n}=180)$ e Virginópolis $(\mathrm{n}=169)$, dando origem às "normas específicas"; (b) não estratificadas - abrangendo, assim, o conjunto das localidades, num total de 993 talhões, originando as "normas gerais".

Em cada localidade (opção a), inicialmente, a população de talhões foi estratificada por idade, em intervalos de 12 meses contados a partir da menor idade. Para cada classe de idade, calcularam-se a média e o desvio-padrão dos dados de incremento médio anual (IMA) do volume de tronco, expresso em m ${ }^{3} \mathrm{ha}^{-1} \mathrm{ano}^{-1}$. A seguir, fez-se a subdivisão da população em talhões de baixa e de alta produtividade,

\section{Quadro 1. Coordenadas geográficas e altitudes das} localidades estudadas

\begin{tabular}{llll}
\hline Localidade & Latitude & Longitude & Altitude \\
\hline Cocais & $19^{\circ} 23^{\prime} 41^{\prime \prime}$ & $42^{\circ} 4^{\prime} 7^{\prime} 11^{\prime \prime}$ & 950 \\
Piracicaba & $19^{\circ} 39^{\prime} 02^{\prime \prime}$ & $43^{\circ} 01^{\prime} 07^{\prime \prime}$ & 880 \\
Rio Doce & $19^{\circ} 09^{\prime} 34^{\prime \prime}$ & $42^{\circ} 25^{\prime} 07^{\prime \prime}$ & 480 \\
Sabinópolis & $18^{\circ} 41^{\prime} 09^{\prime \prime}$ & $42^{\circ} 56^{\prime} 56^{\prime \prime}$ & 880 \\
Santa Bárbara & $20^{\circ} 00^{\prime} 29^{\prime \prime}$ & $43^{\circ} 21^{\prime} 50^{\prime \prime}$ & 820 \\
Virginópolis & $18^{\circ} 40^{\prime} 03^{\prime \prime}$ & $42^{\circ} 30^{\prime} 08^{\prime \prime}$ & 860 \\
\hline
\end{tabular}

sendo esta última a população de referência, definida como sendo maior que a média $+0,5$ desvio-padrão. Quando os 993 talhões foram considerados em conjunto (opção b), a população de referência foi constituída pelos povoamentos classificados como de alta produtividade nas várias localidades e respectivas faixas de idade.

No presente trabalho, as condições de manejo, o material amostrado, a idade e o material genético dos plantios de eucaliptos foram praticamente os mesmos em todas as localidades. Essa similaridade de condições permite verificar se realmente existem diferenças entre normas específicas e gerais, pelo menos em relação ao local de cultivo.

A diagnose do estado nutricional foi efetuada pelos métodos DRIS (Beaufils, 1973) e M-DRIS (Hallmark et al., 1987), sendo consideradas todas as relações (formas diretas e inversas). A diagnose foi também realizada pelo método CND (Diagnose da Composição Nutricional), conforme Parent \& Dafir (1992).

Para o DRIS e o M-DRIS, com os teores dos talhões da população de referência, foram calculadas as relações quocientes duais, incluindo as relações dos nutrientes e da matéria seca, para o M-DRIS. As médias e os desvios-padrão, de todas as relações duais entre os nutrientes considerados, da população de referência, constituem as normas. No cálculo das funções DRIS e M-DRIS (f), as relações quocientes duais são estudentizadas (Alvarez V. \& Leite, 1999), utilizando a fórmula de Jones (1981), da seguinte forma:

$$
\mathrm{f}(\mathrm{A} / \mathrm{B})=10[(\mathrm{~A} / \mathrm{B})-(\mathrm{a} / \mathrm{b})] / \mathrm{s}
$$

em que $10=$ coeficiente multiplicativo de valor arbitrário; $\mathrm{A} / \mathrm{B}=$ relação dual entre as concentrações $\left(\right.$ dag $\mathrm{kg}^{-1}$ ) dos nutrientes A e B do talhão sob diagnose; $\mathrm{a} / \mathrm{b}=$ média das relações duais entre as concentrações (dag $\mathrm{kg}^{-1}$ ) dos nutrientes A e B da subpopulação de referência; e s = desvio-padrão das relações duais da subpopulação de referência.

A partir dos valores de todas as funções DRIS, foram cálculados os índices DRIS, de acordo com a seguinte fórmula:

$$
\begin{aligned}
& I_{A}=\bar{f}=[f(A / B)-f(B / A)+f(A / C)-f(C / A)+ \\
& \ldots \ldots .-f(N / A)] / n
\end{aligned}
$$

em que, $I_{A}=$ índice DRIS do nutriente; $\bar{f}=$ média das funções DRIS (f(A/B) e $\mathrm{f}(\mathrm{B} / \mathrm{A})$ ), funções DRIS nas formas diretas e inversas, respectivamente; e $n$ = número de funções DRIS consideradas.

Posteriormente, somando, em módulo, os índices dos nutrientes e dividindo-se esse valor pelo número de nutrientes envolvidos na análise, obteve-se o índice de equilíbrio nutricional médio $\left(\operatorname{IEN}_{\mathrm{m}}\right)$ (Wadt, 1996), dos talhões das duas populações de interesse. 
Para o cálculo das funções e dos índices M-DRIS, além das relações duais, foram considerados os teores dos nutrientes. Desta forma, de modo análogo à obtenção dos índices DRIS, mediante a média das funções dos teores dos nutrientes envolvidos na diagnose, obteve-se, também, o índice M-DRIS de matéria seca.

Para o método CND, com os teores foliares dos talhões da população de referência, foram calculadas as variáveis multinutrientes $\mathrm{Z}_{\mathrm{i}}(\mathrm{ZN}, \mathrm{ZP}$, $\mathrm{ZK}, \mathrm{ZCa}$ e $\mathrm{ZMg}$ ), de acordo com as seguintes fórmulas (Parent \& Dafir, 1992):

$$
\begin{gathered}
\mathrm{R}=100-\sum \mathrm{x}_{\mathrm{i}} \\
\mathrm{g}(\mathrm{x})=(\mathrm{xN} \cdot \mathrm{xP} \cdot \mathrm{xK} \cdot \mathrm{xCa} \cdot \mathrm{xMg} \cdot \mathrm{R})^{(1 /(\mathrm{D}+1))} \\
\mathrm{Z}_{\mathrm{i}}=\ln \left[\left(\mathrm{x}_{\mathrm{i}} / \mathrm{g}(\mathrm{x})\right]\right.
\end{gathered}
$$

em que $\mathrm{R}=$ valor do complemento para $100 \mathrm{dag} \mathrm{kg}^{-1}$ de matéria seca em relação à soma dos teores dos nutrientes $\mathrm{x}_{\mathrm{i}}(\mathrm{xN}, \mathrm{xP}, \mathrm{xK}, \mathrm{xCa}$ e $\mathrm{xMg})$, em dag $\mathrm{kg}^{-1}$.

$\mathrm{g}(\mathrm{x})=$ média geométrica da composição nutricional (dos teores dos nutrientes, incluindo o complemento $\mathrm{R})$.

$\mathrm{D}$ = número dos nutrientes envolvidos na diagnose; $\mathrm{Z}_{\mathrm{i}}=$ variável multinutriente do nutriente $\mathrm{x}_{\mathrm{i}}$.

As médias $\left(\mathrm{z}_{\mathrm{i}}\right)$ e os desvios-padrão $\left(\mathrm{sz}_{\mathrm{i}}\right)$ constituem as normas da população de referência. No cálculo dos índices $\mathrm{Iz}_{\mathrm{i}}$, para o $\mathrm{CND}$, a variável multinutriente $\mathrm{Z}_{\mathrm{i}}$ é estudentizada, utilizando-se a seguinte fórmula:

$$
\mathrm{Iz}_{\mathrm{i}}=\left(\mathrm{Z}_{\mathrm{i}}-\mathrm{z}_{\mathrm{i}}\right) / \mathrm{sz} \text {, }
$$

em que $\mathrm{Iz}_{\mathrm{i}}=$ índice das variáveis multinutrientes; $\mathrm{Z}_{\mathrm{i}}$ = variável multinutriente da amostra do talhão sob diagnose; $z_{i}=$ média da variável multinutriente da subpopulação de referência, $\mathrm{sz}_{\mathrm{i}}=$ desvio-padrão da variável multinutriente da subpopulação de referência.

Também, para o CND, foi calculado o $\mathrm{IEN}_{\mathrm{m}}$, para os talhões das duas populações de interesse.

O cálculo das funções e dos índices, para os métodos DRIS e M-DRIS, e das variáveis multinutrientes (ZN até $\mathrm{ZMg}$ ) e dos índices, para o método CND, foi efetuado com o auxílio de rotinas desenvolvidas em Excel 5.0.

Para a interpretação dos índices DRIS ou CND ou M-DRIS (Quadro 2), incorporou-se o conceito de potencial de resposta à adubação (PRA) (Wadt, 1996). Foram considerados para o DRIS ou para o CND: o índice, o módulo do índice e se o nutriente é o mais limitante por falta ou por excesso e, para o M-DRIS, o índice M-DRIS ajustado, o módulo do índice e se é o mais limitante por falta ou por excesso. Para tanto, nos talhões de baixa produtividade, os nutrientes, em cada localidade, foram classificados segundo o potencial de resposta à adubação em respostas: positiva (p), positiva ou nula (pz), nula
Quadro 2. Interpretação dos índices DRIS ou CND, ou do índice M-DRIS, na diagnose nutricional de plantios de eucalipto, considerando o potencial de resposta à adubação (PRA)

\begin{tabular}{ccll}
\hline $\begin{array}{c}\text { Índice DRIS ou } \\
\text { CND ou Índice } \\
\text { M-DRIS ajustado }\end{array}$ & $\begin{array}{c}\text { Módulo do } \\
\text { Índice }\end{array}$ & + LF ou + LE & PRA \\
\hline & & & \\
\hline 0 & $>\mathrm{IEN}_{\mathrm{m}}$ & sim & $\mathrm{p}$ \\
$<0$ & $>\mathrm{IEN}_{\mathrm{m}}$ & não & $\mathrm{pz}$ \\
$>0$ & $>\mathrm{IEN}_{\mathrm{m}}$ & sim & $\mathrm{n}$ \\
$>0$ & $>\mathrm{IEN}_{\mathrm{m}}$ & não & $\mathrm{nz}$ \\
$\leq 0$ & $\leq \mathrm{IEN}_{\mathrm{m}}$ & independente & $\mathrm{z}$ \\
$\geq 0$ & $\leq \mathrm{IEN}_{\mathrm{m}}$ & independente & $\mathrm{z}$
\end{tabular}

Índice M-DRIS ajustado = índice do nutriente - índice de matéria seca; $\mathrm{IEN}_{\mathrm{m}}=$ índice de equilíbrio nutricional médio $+\mathrm{LF}=0$ mais limitante por falta; $+\mathrm{LE}=0$ mais limitante por excesso; positiva (p); positiva ou nula (pz); negativa (n); negativa ou nula (nz) e nula (z). Adaptado de Wadt (1996).

(z), negativa ou nula (nz) e negativa (n). Para interpretação dos índices M-DRIS, inicialmente, calculou-se o índice M-DRIS ajustado, que corresponde à diferença entre o índice de determinado nutriente e o índice de matéria seca (IMS).

A universalidade das normas foi avaliada, comparando-se a freqüência de diagnoses concordantes (FDC) do potencial de resposta à adubação (PRA), obtida mediante normas específicas ou gerais, para os métodos DRIS, M-DRIS e CND, contemplando os seguintes critérios de avaliação: os nutrientes separadamente; desde todos (5) até nenhum nutriente (0), e apenas para o limitante primário por deficiência (p) ou por excesso (n).

\section{RESULTADOS E DISCUSSÃO}

A freqüência de diagnoses concordantes (FDC) do potencial de resposta à adubação (PRA) para o conjunto das localidades e para os nutrientes $\mathrm{N}, \mathrm{P}$, $\mathrm{K}, \mathrm{Ca}$ e Mg foi, em média, $85 \%$ (DRIS e M-DRIS) e $84 \%$ (CND) (Quadro 3). Isto indica que, em média, $15 \%$ (DRIS e M-DRIS) e $14 \%$ (CND) dos diagnósticos derivados de normas específicas e de normas gerais foram diferentes.

Avaliou-se também a universalidade das normas por outro critério, em que se comparou a FDC do PRA, derivada de normas específicas ou gerais, desde todos (5) nutrientes até nenhum (0). Constatou-se, em média, por esse critério, para o conjunto das localidades, que $57 \%$ (DRIS), $52 \%$ (M-DRIS) e $53 \%$ (CND) dos talhões apresentaram diagnoses concordantes, ou seja, $43 \%$ (DRIS), $48 \%$ (M-DRIS) 
e $47 \%$ (CND) dos talhões foram diagnosticados diferentemente (Quadro 4). Este critério, sem dúvida, o de maior rigor, resulta, portanto, em menor concordância das diagnoses derivadas de normas específicas ou gerais. Utilizando este critério, não se tem informação sobre a classe de potencial de resposta ( $\mathrm{p}$ e $\mathrm{n}$ ) mais bem diagnosticada. Por isso, adotou-se um terceiro critério com vistas em avaliar

Quadro 3. Freqüência de talhões com diagnoses concordantes do potencial de resposta à adubação( ${ }^{(1)}$ de N, P, K, Ca e Mg, em Eucalyptus grandis, subpopulação de baixa produtividade ${ }^{(2)}$, na região CentroLeste de Minas Gerais, utilizando os métodos DRIS ${ }^{(3)}$, M-DRIS ${ }^{(4)}$ e CND $^{(5)}$, mediante normas específicas para cada localidade e normas gerais para o conjunto das localidades, aplicados em análises de folhas

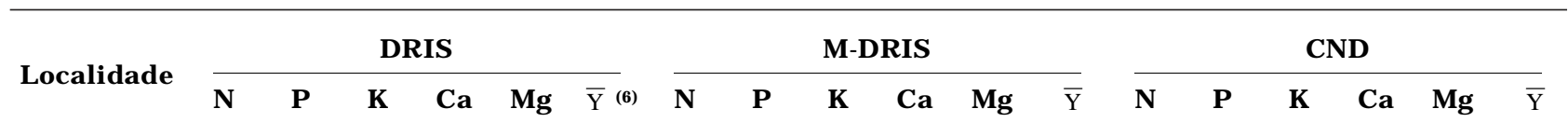

Cocais

( $\mathrm{n}=129)$

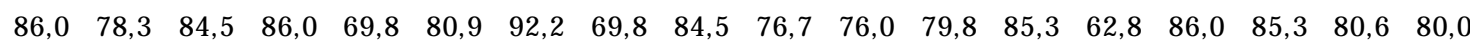

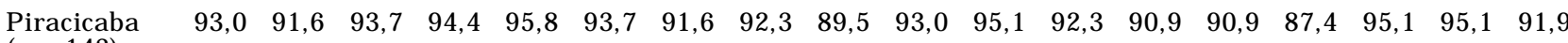
(n = 143)

Rio Doce $(\mathrm{n}=36)$

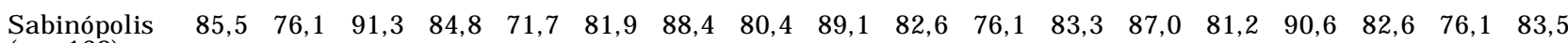
$(\mathrm{n}=138)$

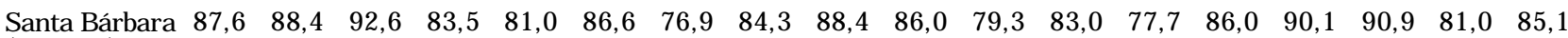
$(\mathrm{n}=121)$

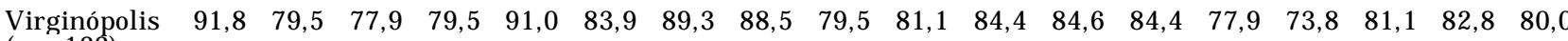
$(\mathrm{n}=122)$

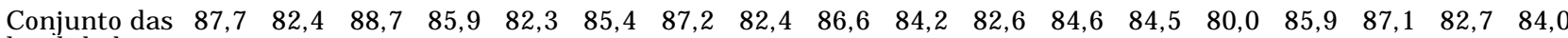
localidades

$(\mathrm{n}=689)$

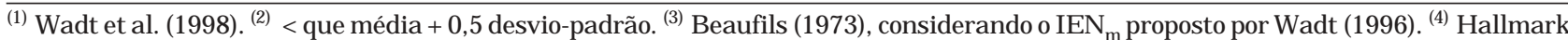
et al. (1987). ${ }^{(5)}$ Parent \& Dafir (1992). ${ }^{(6)}$ Média da frequência dos nutrientes.

Quadro 4. Freqüência de talhões com diagnoses concordantes do potencial de resposta à adubação(1) para os nutrientes considerados concomitantemente conforme as situações $(5,4,3,2,1$ e 0$)$ em Eucalyptus grandis, subpopulação de baixa produtividade ${ }^{(2)}$, na região Centro-Leste de Minas Gerais, utilizando os métodos DRIS ${ }^{(3)}$, M-DRIS ${ }^{(4)}$ e CND $^{(5)}$, mediante normas específicas para cada localidade e normas gerais para o conjunto das localidades aplicados em análises de folhas

\begin{tabular}{|c|c|c|c|c|c|c|c|c|c|c|c|c|c|c|c|c|c|c|}
\hline \multirow{3}{*}{ Localidade } & \multicolumn{6}{|c|}{ DRIS } & \multicolumn{6}{|c|}{ M-DRIS } & \multicolumn{6}{|c|}{ CND } \\
\hline & \multicolumn{18}{|c|}{ Nutrientes diagnosticados concomitantemente ${ }^{(6)}$} \\
\hline & 5 & 4 & 3 & 2 & 1 & $\mathbf{0}$ & 5 & 4 & 3 & 2 & $\mathbf{1}$ & $\mathbf{0}$ & 5 & 4 & 3 & 2 & $\mathbf{1}$ & $\mathbf{0}$ \\
\hline $\begin{array}{l}\text { Cocais } \\
(\mathrm{n}=129)\end{array}$ & 44,2 & 24,0 & 24,8 & 6,2 & 0,8 & 0,0 & 38,0 & 32,6 & 20,9 & 7,8 & 0,8 & 0,0 & 44,2 & 24,8 & 21,7 & 5,4 & 3,9 & $\overline{0,0}$ \\
\hline $\begin{array}{l}\text { Piracicaba } \\
(\mathrm{n}=143)\end{array}$ & 79,7 & 11,9 & 5,6 & 2,8 & 0,0 & 0,0 & 70,6 & 20,3 & 9,1 & 0,0 & 0,0 & 0,0 & 73,4 & 15,4 & 9,1 & 1,4 & 0,7 & 0,0 \\
\hline $\begin{array}{l}\text { Rio Doce } \\
(\mathrm{n}=36)\end{array}$ & 47,2 & 25,0 & 25,0 & 0,0 & 2,8 & 0,0 & 50,0 & 22,2 & 16,7 & 8,3 & 2,8 & 0,0 & 38,9 & 30,6 & 19,4 & 11,1 & 0,0 & 0,0 \\
\hline $\begin{array}{l}\text { Sabinópolis } \\
(\mathrm{n}=138)\end{array}$ & 47,1 & 23,2 & 23,2 & 5,1 & 1,4 & 0,0 & 47,1 & 30,4 & 15,2 & 6,5 & 0,7 & 0,0 & 47,8 & 30,4 & 14,5 & 5,8 & 1,4 & 0,0 \\
\hline $\begin{array}{l}\text { Santa Bárbara } \\
(\mathrm{n}=121)\end{array}$ & 60,3 & 18,2 & 16,5 & 4,1 & 0,8 & 0,0 & 50,4 & 21,5 & 21,5 & 5,8 & 0,8 & 0,0 & 55,4 & 24,0 & 14,0 & 4,1 & 2,5 & 0,0 \\
\hline $\begin{array}{l}\text { Virginópolis } \\
(\mathrm{n}=122)\end{array}$ & 51,6 & 23,0 & 21,3 & 1,6 & 2,5 & 0,0 & 53,3 & 26,2 & 12,3 & 6,6 & 1,6 & 0,0 & 44,3 & 23,8 & 15,4 & 2,8 & 5,6 & 0,0 \\
\hline $\begin{array}{l}\text { Conjunto das } \\
\text { localidades } \\
(\mathrm{n}=689)\end{array}$ & 56,5 & 20,2 & 18,4 & 3,8 & 1,2 & 0,0 & 52,1 & 26,0 & 15,7 & 5,4 & 0,9 & 0,0 & 52,7 & 23,9 & 15,1 & 4,3 & 2,6 & 0,0 \\
\hline
\end{tabular}


a universalidade das normas. Por este critério, verificou-se a FDC do PRA para o limitante primário por deficiência (resposta "p") e para o limitante primário por excesso (resposta "n"). A idéia é que é mais importante haver concordância para este tipo de resposta, uma vez que ela permitiria a correção mediante adição do respectivo nutriente. Considerando o conjunto das localidades, a FDC do PRA foi: para resposta "p": $85 \%$ (DRIS), $82 \%$ (MDRIS) e $83 \%$ (CND); e, para resposta "n": $86 \%$ (DRIS), $76 \%$ (M-DRIS) e $83 \%$ (CND) (Quadro 5).

A FDC do PRA, avaliada pelos três critérios, mostrou-se dependente das localidades. As localidades Piracicaba e Cocais (DRIS e M-DRIS), Piracicaba e Rio Doce (CND) apresentaram a maior e a menor concordância, para os dois primeiros critérios; enquanto, para o terceiro critério, foram Piracicaba e Rio Doce, respectivamente.

Nos diferentes critérios utilizados para avaliar a universalidade dos métodos DRIS, M-DRIS e CND, é importante enfatizar que as comparações dos

Quadro 5. Freqüência de talhões com diagnoses concordantes do potencial de resposta à adubação $^{(1)}$ (p e n) em Eucalyptus grandis, subpopulação de baixa produtividade ${ }^{(2)}$, na região Centro-Leste de Minas Gerais, utilizando os métodos DRIS ${ }^{(3)}$, M-DRIS ${ }^{(4)}$ e $\mathrm{CND}^{(5)}$, mediante normas específicas para cada localidade e normas gerais para o conjunto das localidades aplicados em análises de folhas

\begin{tabular}{llll}
\hline Localidade & Método & p & $\mathbf{n}$ \\
\hline Cocais & DRIS & 84,5 & 80,6 \\
(n=129) & M-DRIS & 79,0 & 66,0 \\
& CND & 84,5 & 74,6 \\
Piracicaba & DRIS & 92,3 & 94,3 \\
(n=143) & M-DRIS & 88,2 & 84,6 \\
& CND & 92,0 & 91,7 \\
Rio Doce & DRIS & 69,4 & 91,7 \\
(n=36) & M-DRIS & 78,6 & 70,8 \\
& CND & 75,0 & 82,9 \\
Sabinópolis & DRIS & 86,9 & 76,6 \\
(n=138) & M-DRIS & 82,8 & 68,3 \\
& CND & 79,4 & 84,5 \\
Santa Bárbara & DRIS & 83,3 & 88,7 \\
(n=121) & M-DRIS & 79,8 & 78,0 \\
& CND & 83,1 & 85,3 \\
Virginópolis & DRIS & 83,6 & 85,3 \\
(n=122) & M-DRIS & 81,7 & 81,7 \\
& CND & 77,7 & 76,1 \\
Conjunto das & DRIS & 85,4 & 85,5 \\
localidades & M-DRIS & 82,3 & 75,5 \\
(n=689) & CND & 83,1 & 82,7 \\
& & & \\
& & &
\end{tabular}

${ }^{(1)}$ Wadt et al. (1998). ${ }^{(2)} \leq$ que média + 0,5 desvio-padrão. ${ }^{(3)}$ Beaufils (1973), considerando o IEN $_{m}$ proposto por Wadt et al. (1998). ${ }^{(4)}$ Hallmark et al. (1987). ${ }^{(5)}$ Parent \& Dafir (1992); $p=$ limitante primário por deficiência; $n$ = limitante primário por excesso. diagnósticos derivados de normas específicas ou gerais foram realizadas com os dados de cada um dos talhões, diferentemente das comparações realizadas na maioria dos trabalhos relacionados sobre este tema, nos quais se comparam os valores das percentagens de talhões diagnosticados como deficientes, equilibrados ou em excesso, desconsiderando o fato de que os respectivos diagnósticos podem ser originados de diferentes talhões, o que acarretaria equívocos.

A análise dos valores de FDC, conforme os três grupos de critérios comentados, indica que as diferenças encontradas entre normas específicas e gerais dependem da forma de comparação entre elas. Isso levaria à conclusão de ser o grau de universalidade das normas dependente do critério adotado para sua avaliação.

Portanto, por meio de quaisquer dos critérios adotados, constata-se que há divergência entre os diagnósticos derivados de normas específicas ou gerais para o eucalipto, indicando, desta forma, ausência de universalidade das normas, em oposição à idéia comumente exposta na literatura (Sumner, 1979; Walworth \& Sumner, 1987; Snyder \& Kretschmer, 1988; Snyder et al., 1989; Payne et al., 1990; Wadt, 1996; Bailey et al., 1997).

A habilidade do DRIS em identificar diferenças no status de nutrientes não pode servir como justificativa para utilização de normas geradas a partir de um amplo banco de dados (normas gerais). Utilizando essas normas, obtêm-se diagnósticos incorretos, ou menos acurados, do que aqueles obtidos a partir de normas específicas, como evidenciado neste trabalho.

Ainda, a literatura registra que o uso de normas DRIS, derivadas de outros locais, só é adequado quando os teores de nutrientes nas lavouras forem semelhantes aos das lavouras sob diagnose (Walworth \& Sumner 1987; Reis Jr. \& Monnerat, 1998). Tal afirmativa é contrariada neste trabalho. As normas pouco variam entre locais (dados não apresentados) e, não obstante, em média, $15 \%$ dos diagnósticos derivados de normas específicas obtidos pelos métodos DRIS, M-DRIS e CND foram diferentes daqueles derivados de normas gerais.

\section{CONCLUSÕES}

1. O grau de universalidade das normas, obtidas em acordo com os respectivos métodos: DRIS, M-DRIS e CND, foi dependente do critério adotado para sua avaliação, variando, também, entre as localidades.

2. Em virtude da ausência de universalidade das normas, é preferível a utilização de normas específicas em vez de normas gerais. 


\section{LITERATURA CITADA}

AITCHISON, J. The statistical analysis of compositional data. J. Royal Stat. Soc. B., 44:139-177, 1982.

ALVAREZ V., V.H. \& LEITE, R.A. Fundamentos estatísticos das fórmulas usadas para cálculos dos índices DRIS. B. Inf. SBCS, 24(1):20-24, 1999.

BAILEY, J.S.; BEATTIE, J.A.M. \& KILPATRICK, D.J. The diagnosis and recommendation integrated system (DRIS) for diagnosing the nutrient status of grassland swards: I. Model establishment. Plant Soil, 197:127-135, 1997.

BEAUFILS, E.R. Diagnosis and recommendation integrated system (DRIS). Pietermaritizburg, University of Natal, Pietermaritizburg, South Africa, 1973. 131p. (Soil Science Bulletin, 1)

COSTA, A.N. Sistema integrado de diagnose e recomendação (DRIS). B. Inf. SBCS, 24:13-15, 1999.

DARA, S.T.; FIXEN, P.E. \& GELDERMAN, R.H. Sufficiency level and Diagnosis and Recommendation Integrated System approaches for evaluating the nitrogen status of the corn. Agron. J., 84:1006-1010, 1992.

ESCANO, C.R.; JONES, C.A. \& UEHARA, G. Nutrient diagnosis in corn on Hydric Dystrandepts : II. Comparison of two systems of tissue diagnosis. Soil Sci. Soc. Am. J., 45:1140$1144,1981$.

HALLMARK, W.B.; WALWORTH, J.L.; SUMNER, M.E.; MOOY, C.J.; PESEK, J. \& SHAO, K.P. Separating limiting and non-limiting nutrients. J. Plant. Nutr., 10:1381-1390, 1987.

HOLLAND, D.A. The interpretation of leaf analysis. J. Hortic. Sci., 41:311-329, 1966.

JONES, C.A. Proposed modifications of the Diagnosis and Recomendation Integrated System (DRIS) fo interpreting plant analysis. Comm. Soil Sci. Plant Anal., 22:785-794, 1981.
JONES Jr., J. Modern interpretation systems for soil and plant analysis in the USA. Aus. J. Exp. Agric., 33:1039-1043, 1993.

PARENT, L.E. \& DAFIR, M. A theoretical concept of Compositional Nutrient Diagnosis. J. Am. Soc. Hortic. Sci., 117:239-242, 1992.

PAYNE, G.G.; RECHCIGL, J.E. \& STEPHENSON, R.L. Development of Diagnosis and Recommendation Integrated System norms for Bahiagrass. Agron. J., 82:930-934, 1990.

REIS Jr., R.A. \& MONNERAT, P.H. Diagnose nutricional da cana-de-açúcar através do sistema integrado de diagnose e recomendação (DRIS). In: REUNIÃO BRASILEIRA DE FERTILIDADE DO SOLO E NUTRIÇÃO DE PLANTAS, 23., Caxambú, 1998. Anais. Caxambú, 1998. p.751.

REIS Jr., R.A. Universalidade das normas DRIS na cultura do milho. In: CONGRESSO BRASILEIRO DE CIÊNCIA DO SOLO, 28., Londrina, 2001. Anais. Londrina, Sociedade Brasileira de Ciência do Solo, 2001. p.195.

SNYDER, G.H. \& KRETSCHMER Jr, A.E. A DRIS analysis for Bahiagrass pastures. Soil Crop Sci. Soc. Florida Proc., 47:5659, 1988.

SNYDER, G.H.; SANCHEZ, C.A. \& ALRIGHTS, J.S. DRIS evaluation of the nutrient status of Bahia and St. Augustine turfgrasses. Proc. Florida State Hortic. Soc., 102:133-137, 1989.

SUMNER, M.E. Interpretation of foliar analysis for diagnostic purposes. Agron. J., 71:343-348, 1979.

WADT, P.G.S. Os métodos da chance matemática e do Sistema Integrado de Diagnose e Recomendação (DRIS) na avaliação nutricional de plantios de eucalipto. Viçosa, Universidade Federal de Viçosa, 1996. 123p. (Tese de Doutorado)

WALWORTH, J.L. \& SUMNER, M.E. The diagnosis and recommendation integrated system (DRIS). Adv. Soil Sci., 6:149-188, 1987

WORTMANN, C.S.; KISAKYE, J. \& EDJE, O.T. The diagnosis and recommentation integrated system for dry bean: determination and validation of norms. J. Plant Nutr., 15:2369-2379, 1992. 